\title{
Clinical Evaluation of Semi-Adjustable Articulators: Reproducibility of Sagittal Condylar Path Inclination Assessed by a Jaw-Tracking System with Six Degrees of Freedom
}

\author{
Katsuomi Hangai, DDS, ${ }^{a}$ Kumiko Aridome, DDS, PhD, ${ }^{b}$ Chau-Hsiang Wang, DDS, PhD, ${ }^{c}$ \\ and Yoshimasa Igarashi, DDS, $\mathrm{PhD}^{\mathrm{b}}$ \\ a Igari Dental Clinic, Iwaki, Fukushima, Japan \\ ${ }^{b}$ Removable Partial Denture Prosthodontics, Department of Masticatory Function Rehabilitation, Division of Oral \\ Health Sciences, Graduate School, Tokyo Medical and Dental University, Tokyo, Japan \\ c School of Dentistry, Kaohsiung Medical Collage, Kaohsiung, Taiwan
}

\section{Clinical significance}

This study evaluated the reproducibility of sagittal condylar path inclination with seven types of semi-adjustable articulators used in clinical settings.

\begin{abstract}
Purpose: To validate whether the data for individual patients are correctly reproduced in sagittal condylar path inclination of the articulator, the data obtained by the conventional anterior check bite method and the data obtained by a jaw-tracking system with six degrees of freedom were compared.

Methods: In 5 subjects (4 males and 1 female) with healthy tooth alignments, 5 anterior check bites were obtained from each subject. Sagittal condylar path inclination was measured using seven types of semi-adjustable articulator. Next, the anterior gliding movement was measured 5 times in all 5 subjects using a jaw-tracking system with six degrees of freedom (MMJI-E, Shofu Inc. Kyoto, Japan. The sagittal condylar path inclination data obtained with semi-adjustable articulators were compared to those obtained by the jawtracking system using the two-way analysis of variance and Fisher's PLSD method ( $\alpha=0.05)$.

Results: In measurements of the same subjects for five types of articulators with the check bite method, significant differences were observed by two-way analysis of variance, and differences in measurements among articulators were found $(p<0.01)$.

Regarding the sagittal condylar path inclinations measured with Hanau-184 Wide-View and Dentatus ARL

Corresponding to: Dr Kumiko Aridome

Removable Partial Denture Prosthodontics,

Department of Masticatory Function Rehabilitation,

Division of Oral Health Sciences, Graduate School,

Tokyo Medical and Dental University

1-5-45 Yushima, Bunkyo-ku, Tokyo 113-8549, Japan

Tel: +81-3-5803-5516, Fax: +81-3-5803-5516

E-mail: arirpro@tmd.ac.jp
\end{abstract}

Received on June 6, 2007/Accepted on November 30, 2007 articulators, significant differences were observed on both right and left sides compared with the jaw-tracking system. With the Denar Centri-Check System and Denar Mark II, significant differences were observed on one side $(p<0.05)$.

Conclusion: Denar Cadiax Compact, Hanau Condyle Repositioner, and Hanau $\mathrm{H} 2 \mathrm{O}$ are recommended for more accurate measurements of the sagittal condylar path inclination.

Key words: semi-adjustable articulators, sagittal condylar path inclination, inclination of sagittal condylar path, jaw movement with six degrees of freedom, jaw ${ }^{-}$ tracking system

\section{Introduction}

Semi-adjustable articulators are used both to diagnose occlusion at protruded and lateral eccentric jaw positions and to select the optimal treatment plan. In addition, based on the reproduced jaw movement, the arrangement of artificial teeth and the fabrication of prosthetic appliances are also performed. Semi-adjustable articulators have multiple advantages over the average value types. They have mechanical condylar elements which can be adjusted for lateral and descending movements of the mandible by positional interocclusal records, ${ }^{1}$ although the condylar path is reproduced linearly. The maxillary cast is mounted at the appropriate relationship to the condylar hinge axis using a face-bow transfer. ${ }^{2}$ The articulator is approximately the same size as the temporomandibular joint in humans and it minimizes the errors related to the hinge axis radius and intercondylar distance. ${ }^{2}$

When determining the sagittal condylar path inclinations using clinical procedures, the anterior 
Table 1 Articulator systems used in this study.

\begin{tabular}{lllll}
\hline Articulator & Manufacture & Code & Type & Purpose \\
\hline Hanau Condyle Repositioner 181 & HANAU & 181 & Condylar types & For diagnosis \\
Hanau Wide-View 184 & HANAU & 184 & Condylar types & For dental techniques \\
Hanau H2O & HANAU & H2O & Condylar types & For dental techniques \\
Dentatus ARL & DENTATUS & ARL & Condylar types & For dental techniques \\
Denar Centri-Check System & DENAR & DCS & Arcon types & For diagnosis \\
Denar Mark II & DENAR & MK II & Arcon types & For dental techniques \\
Denar Cadiax Compact & GAMMA & CDX & Arcon types & For diagnosis \\
\hline
\end{tabular}

check bite method is preferred as the usual method for reproducing them in a semi-adjustable articulator. However, it has often been suggested that the sagittal condylar path inclinations obtained by this method are unstable. ${ }^{3}$

Gross et al. $^{3}$ determined the horizontal reference plane by cephalogram and adjusted the sagittal condylar inclination of three types of semiadjustable articulators by the check bites of the same multiple patients to compare the results. dos Santos Jr. ${ }^{4}$ measured the mandibular movement using Cyberhoby computer pantographs of three subjects and the movements of six types of articulators. However, no studies exist that compare many types of semi-adjustable articulators to actual sagittal condylar inclination by utilizing accurate jaw-tracking systems.

Recently, various jaw-tracking systems with six degrees of freedom have been developed that can accurately measure mandibular movements ${ }^{5-8}$ and thus provide a visual evaluation of jaw movement pathways and multi-variable analysis of numerical jaw movement parameters. They are also clinically useful for diagnosing temporomandibular disorders and making dental prostheses. ${ }^{8}$ Furthermore, a novel robotic articulator that reproduced a six-degree-of-freedom jaw movement was developed and it has been demonstrated to be a useful device. $^{9}$

The purpose of this study was to evaluate whether the sagittal condylar inclination for individual patients is correctly reproduced in the leading seven types of semi-adjustable articulators by comparing the data obtained by a jaw-tracking system with six degrees of freedom.

\section{Materials and Methods \\ Subjects}

The subjects consisted of five members of the medical staff at the First Department of Prosthetic
Dentistry, Matsumoto Dental University (4 males and 1 female), with a mean age of 31 years. Measurements were taken after explaining the method of the experiment to all the subjects and obtaining their informed consent.

The inclusion criteria included: those with individual normal occlusion without loss of teeth, except for third molar, with stable intercuspal relations. The exclusion criteria included: those with edge-to-edge occlusion or an anterior cross-bite; an acute condition in the maxillofacial region; jaw arthritis; being in the midst of treatment for jaw functional abnormalities; and tooth mobility of 2 or more.

\section{Measurement of sagittal condylar path angles utilizing the anterior check bite method}

From each subject, alginate impressions (Algiace, Densply Sankin, Tokyo, Japan) of the maxillary and mandibular arches were taken and filled with dental superhard plaster (New Fujirock, GC, Tokyo, Japan). The powder-water ratio was set at 0.2 according to the manual. The maxillary cast was mounted with hard plaster (Himaunt, San-esu, Osaka, Japan) in each semi-adjustable articulator using the corresponding face-bow. The mandibular cast was then mounted in intercuspal position without any registration, while fitting the cast together. As for the anterior check bite, each subject gave an anterior edge-to-edge position five times, and 5 check bites were obtained using vinyl silicon (MEMOREG2, Heraeus Kulzer Japan, Tokyo, Japan).

Seven types of semi-adjustable articulators were used as shown in Table 1 (Figs. 1 and 2). To standardize the base plane of each articulator as the Frankfort horizontal $(\mathrm{FH})$ plane, the infraorbital point was used for the anterior reference point. The ear-piece type face-bow was used for Denar Cadiax Compact (CDX), Denar Centri-Check System (DCS), Denar Mark II (MK II) and a point 13 $\mathrm{mm}$ anterior from the antilobium was set as a rear 


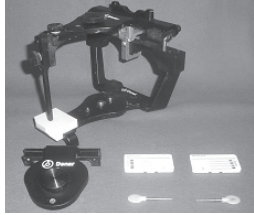

Denar Centri-Check System (DCS)

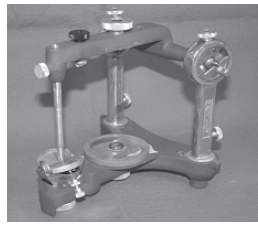

Hana H2O (H2O)

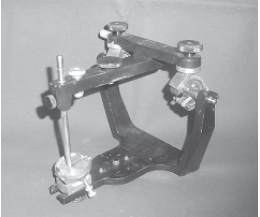

Hanau Wide-View 184 (184)

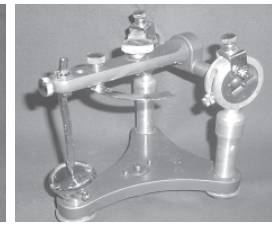

Dentatus ARL (ARL)

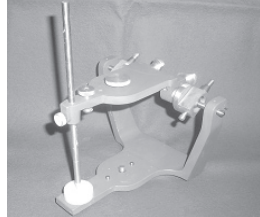

Hanau Condyle

Repositioner 181 (181)

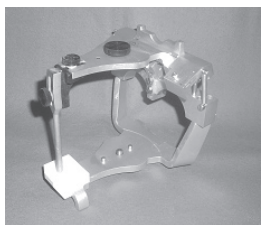

Denar Mark II (MK II)
Fig. 1 Hanau Condyle Repositioner (181), Hanau WideView184 (184), Hanau H2O (H2O), Dentatus ARL (ARL), Denar Centri-Check System (DCS), Denar Mark II (MKII).
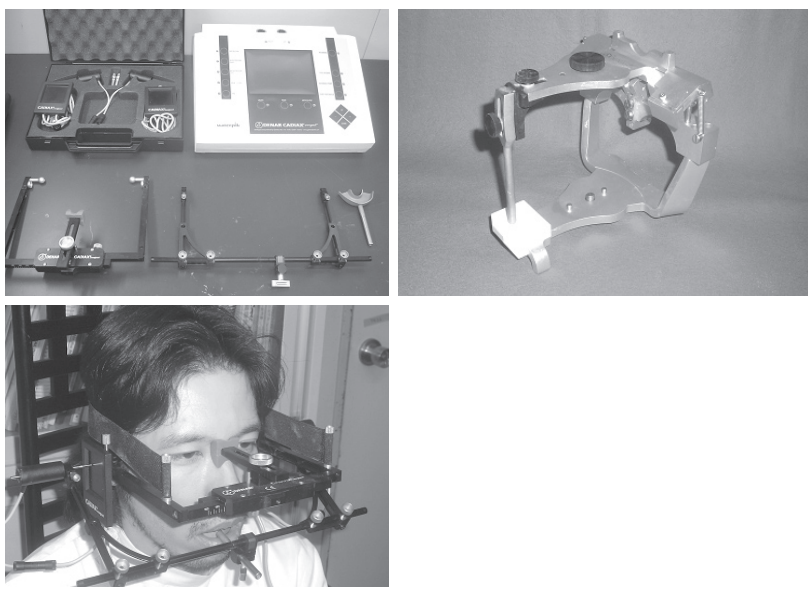

Fig. 2 Denar Cadiax Compact (CDX).

reference point for Hanau Wide-View184 (184), Dentatus ARL (ARL), Hanau Condyle Repositioner (181), and Hanau H2O (H2O). Using the 5 anterior check bites obtained from each subject, left and right sagittal condylar path inclinations were measured partly by the method of Lauritzen..$^{10,11}$ All measurements were taken in accordance with the instructions for all articulators by an experienced dentist.

\section{Measurement of the sagittal condylar inclination by a jaw movement analyzer with six degrees of freedom}

Using a digital type jaw movement analyzer (MMJI-E, Shofu Inc. Kyoto, Japan; Fig. 3), anterior gliding movements were performed 5 times in each subject. The sagittal condylar inclination was

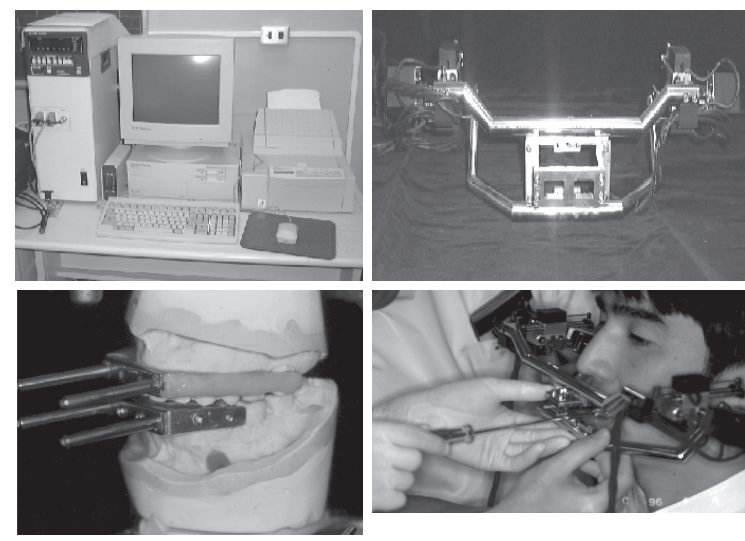

Fig. 3 MM-JI-E (MM).

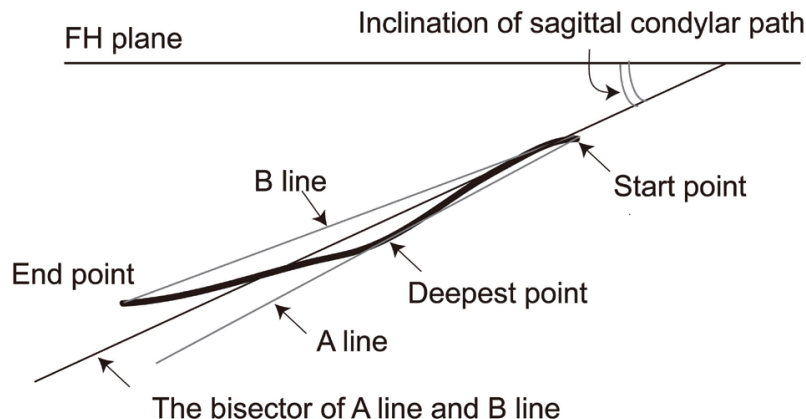

Fig. 4 Schematic illustration of the sagittal condylar path. A line is formed by lines connecting the start point and the deepest point. $\mathrm{B}$ line is formed by the line connecting the start point and the end point. The angle formed by $\mathrm{FH}$ plane and the bisector of A line and B line was defined as sagittal condylar path inclination.

measured in every session of the anterior mandibular movement in sagittal plane displayed on the computer. This analyzer is a jaw-tracking device that measures jaw movement with six degrees of freedom and the spatial accuracy at the incisal point has been reported to be within 0.3 $\mathrm{mm}^{7}$

Since the sagittal condylar path forms a downward convex arch, the angle formed by the bisector of the line linking the start point and the end point and the line linking the start point and the deepest point of the arch and $\mathrm{FH}$ plane was defined as the sagittal condylar inclination (Fig. 4). The representative point of the caput mandibulae used for measurement was defined as the axis point for all movement. 
Table 2 Right: Two-Way Analysis of Variance of sagittal condylar path inclination.

\begin{tabular}{lrcrcc}
\hline Source & $d f$ & $\begin{array}{c}\text { Sum of } \\
\text { squares }\end{array}$ & $\begin{array}{c}\text { Mean- } \\
\text { squares }\end{array}$ & F & $p$ \\
\hline A: Subject & 4 & 20908.234 & 5227.059 & 1061.13 & $* * 5.13 \mathrm{E}-11$ \\
B: Articulator & 7 & 578.604 & 82.658 & 16.781 & $* * 1.65 \mathrm{E}-16$ \\
AXB & 28 & 4663.44 & 166.552 & 33.811 & $* * 2.25 \mathrm{E}-53$ \\
Error & 160 & 788.148 & 4.926 & & \\
\hline
\end{tabular}

$* * p<0.01$

Table 3 Left: Two-Way Analysis of Variance of sagittal condylar path inclination.

\begin{tabular}{lrcrcc}
\hline Source & $d f$ & $\begin{array}{c}\text { Sum of } \\
\text { squares }\end{array}$ & $\begin{array}{c}\text { Mean- } \\
\text { squares }\end{array}$ & F & $p$ \\
\hline A: Subject & 4 & 17977.46 & 4494.37 & 987.095 & $* 1.34 \mathrm{E}-111$ \\
B: Articulator & 7 & 421.25 & 60.178 & 13.217 & $* 2.18 \mathrm{E}-13$ \\
AXB & 28 & 4212.25 & 150.437 & 33.041 & $* 1.04 \mathrm{E}-52$ \\
Error & 160 & 728.5 & 4.553 & & \\
\hline \multicolumn{7}{c}{} & & & & $*$ \\
& & & & &
\end{tabular}

Table 4 Right: Results of multiple comparison test of sagittal condylar path inclination (Fisher's PLSD Method). Asterisk indicates significant difference.

\begin{tabular}{lccc}
\hline & Mean deviation & Reject value & $p$ value \\
\hline MM-181 & -1.50 & 4.16521 & 0.34944 \\
MM-184 & -4.90 & 4.16521 & $* * 0.00253$ \\
MM-H2O & -2.03 & 4.16521 & 0.15209 \\
MM-ARL & -4.02 & 4.16521 & ${ }^{*} 0.01285$ \\
MM-DCS & -3.14 & 4.16521 & 0.05116 \\
MM-MK II & -3.50 & 4.16521 & $* 0.02995$ \\
MM-CDX & -0.04 & 4.16521 & 0.98206 \\
\hline \multicolumn{2}{c}{$* p<0.05,{ }^{* *} p<0.01$}
\end{tabular}

\section{Statistics}

A two-way analysis of variance was performed for both the left and right sagittal condylar path inclinations obtained with the seven types of semi-adjustable articulators and condylar path inclinations obtained with a jaw-tracking system with six degrees of freedom in 5 subjects. In a subsequent analysis, they were compared using Fisher's PLSD method. The significance level was set at $5 \%$.

In addition, a one-way analysis of variance was conducted to compare the articulators of the condylar types (184, ARL, 181, H2O) and the arcon types (CDX, DCS, MK II).

For the statistical analysis, a statistical software program (SPSS version 10J; SPSS Japan Inc, Tokyo, Japan) was used.

\section{Results}

Significant differences were observed in measurements of sagittal condylar inclination with the same
Table 5 Left: Results of multiple comparison test of sagittal condylar path inclination (Fisher's PLSD Method).

\begin{tabular}{lccr}
\hline & Mean deviation & Reject value & \multicolumn{1}{c}{ P value } \\
\hline MM-181 & -1.74 & 3.943233 & 0.25191 \\
MM-184 & -5.06 & 3.943233 & $* * 0.00102$ \\
MM-H2O & -2.42 & 3.943233 & 0.11171 \\
MM-ARL & -3.58 & 3.943233 & ${ }^{*} 0.01915$ \\
MM-DCS & -3.10 & 3.943233 & $* 0.04209$ \\
MM-MK II & -2.94 & 3.943233 & 0.05374 \\
MM-CDX & -1.18 & 3.943233 & 0.43668 \\
\hline
\end{tabular}

${ }^{*} p<0.05,{ }^{* *} p<0.01$

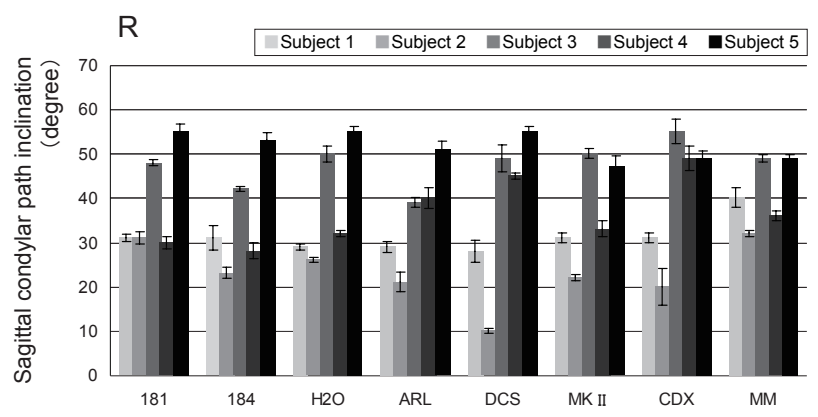

Fig. 5 Sagittal condylar path inclinations measured by each system in Subjects 1-5 (Right). Error bar indicates standard deviation.

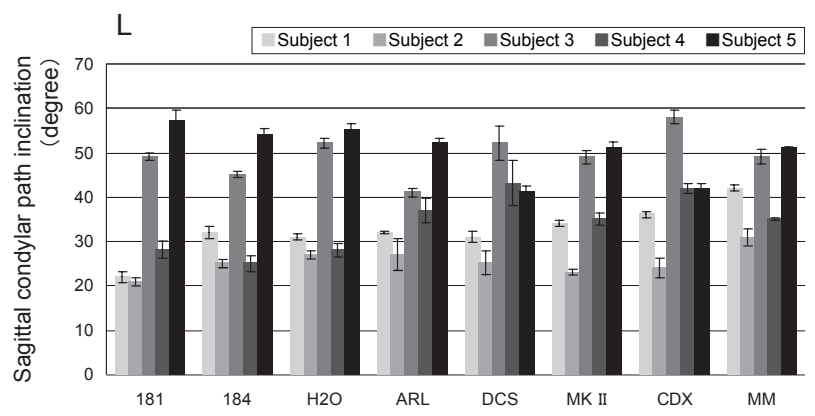

Fig. 6 Sagittal condylar path inclinations measured by each system in Subjects 1-5 (Left). Error bar indicates standard deviation.

subjects in seven types of articulators using the check bite method as a result of two-way analysis of variance and among articulators there were differences in measurements $(p<0.01)$ (Tables 2 and 3 ).

As a result of multiple comparisons, regarding the sagittal condylar path inclinations obtained with 184 and ARL, significant differences from those obtained by the jaw-tracking system with six degrees of freedom were observed on both right and left sides. In addition, in DCS and MK II, significant differences were observed on one side. Moreover, no significant differences were observed between CDX, 181 and H2O (Tables 4 and 5, Figs. $5-8)$. A one-way analysis of variance was conducted comparing the articulators of the condylar types 


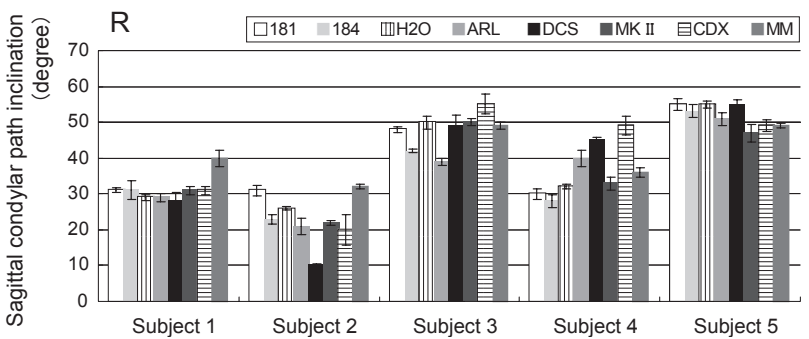

Fig. 7 Sagittal condylar path inclinations measured in each subject by 7 articulators and MM (Right). Error bar indicates standard deviation.

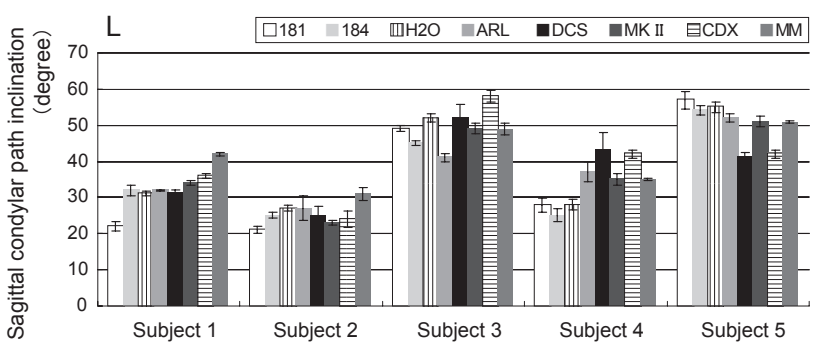

Fig. 8 Sagittal condylar path inclinations measured in each subject by 7 articulators and MM (Left). Error bar indicates standard deviation.

(184, ARL, 181, H2O) and the arcon types (CDX, DCS, MK II). As a result, no significant difference was found $(p<0.05)$ (Figs. 9 and 10).

In addition, the values of the sagittal condylar path inclinations in every articulator were smaller than the actual values (MM) by 0.04 to $5.06 \mathrm{de}^{-}$ grees (Tables 4 and 5).

\section{Discussion}

Among the seven types of articulators used in this study, CDX, 181, and $\mathrm{H} 2 \mathrm{O}$ exhibited superior reproducibility in terms of accurate measurements of sagittal condylar path inclinations. These 3 types of articulator are recommended in the anterior check bite method.

dos Santos ${ }^{4}$ investigated the differences in linear and angular path of different semi-adjustable articulators, which were protrusive and sagittal inclinations, immediate and progressive sideshift, Bennetto angle, then reported that arcon types have higher reproducibility than the condylar types. However, in this study, there were no differences in the sagittal condylar path inclinations with different types of articulators such as the condylar types and the arcon types.

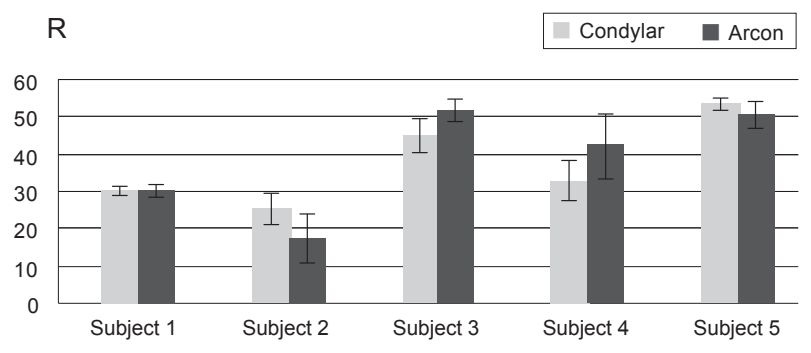

Fig. 9 Sagittal condylar path inclinations in each subject by the condylar type and the arcon type (Right). Error bar indicates standard deviation.

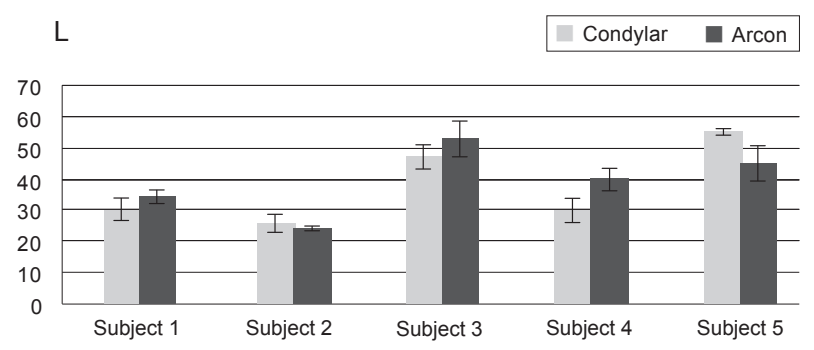

Fig. 10 Sagittal condylar path inclinations in each subject by the condylar type and the arcon type (Left). Error bar indicates standard deviation.

A remarkable feature of this study over previous studies was that the jaw-tracking system with six degrees of freedom, MM-JI-E, was used for measuring actual sagittal condylar inclination. The accuracy of this system depends on the measuring point, that at the original point in the measuring device is within $0.017 \mathrm{~mm}$ and that at the incisal point is within $0.3 \mathrm{~mm} .{ }^{7}$ Since a check bite method is based on a linear relationship of the orbit between the intercuspal and excentric positions during mandibular movement, the condylar paths in semi-adjustable articulators are expressed as linear, thus the condylar path in a human body is represented in a linear manner with this method. In fact, it has been reported that the sagittal condylar inclination reproduced in semi-adjustable articulators with the check bite method tends to be gentler than the condylar inclination because $92 \%$ of the sagittal condylar inclination forms a downward convex arch. ${ }^{12}$ In this study, the values of the sagittal condylar path inclinations in each articulator group were smaller than the actual values by $0.04-5.06$ degrees, and it was similar to the results reported by Aull et al. ${ }^{12}$

One limitation of this study is the different types of articulators such as the condylar types and the arcon types were compared, which all 
have different base planes and rear reference points. To solve this problem, this study standardized the base plane of each articulator as the $\mathrm{FH}$ plane as described above. In this study, no significant difference was found between the two types. However, the sample size was small and it cannot be concluded that these two types have the same accuracy in measuring the sagittal condylar path inclination.

\section{Conclusion}

Among the seven types of articulators used in this study, CDX, 181, and $\mathrm{H} 2 \mathrm{O}$ represented superiority in measuring the sagittal condylar path inclinations. There were no differences between the values obtained by the condylar types and the arcon types. Sagittal condylar path inclination reproduced in semi-adjustable articulators with the check bite method was represented as smaller than the actual values by $0.04-5.06$ degrees.

\section{References}

1. Ash MM, Ranfjord S. Occlusion. Fourth edition. Philadelphia: WB Saunders; 1995, p. 410-417.

2. Mohl ND, Zarb GA, Carlsson GE. A textbook of occlusion. London: Quintessence Publishing; 1988, p. 138141.

3. Gross M, Nemcovsky C, Friedlander LD. Compara- tive study of condylar settings of three semiadjustable articulators. Int J Prosthodont 1990; 3: 135-141.

4. dos Santos J Jr, Ash MM. A comparison of the equivalence of jaw and articulator movements. J Prosthet Dent 1988; 59: 36-42.

5. Huang BY, Durrant CJ, JohnsonCW, Murray GM. A method of indirect registration of the coordinates of condylar points with a six-degree-of-freedom jaw tracker. J Neurosci Methods 2002; 117: 183191.

6. Wakimoto Y, Takamura A, Tokiwa H et al. Development of a robot $(6 \mathrm{DOF} / \mathrm{R} 0)$ for simulation of the mandibular movement with six degrees of freedom. J Jpn Soc Stomatognath Funct 2001; 7: 1-11.

7. Nishigawa K, Satsuma T, Shigemoto S, Bando E, Nakano M, Ishida O. Development of a novel articulator that reproduced jaw movement with six-degree-of-freedom. Med Eng Phys 2007; 29: 615-619.

8. Miyawaki S, Tanimoto Y, Kawakami T, Sugimura M, Takano-Yamamoto T. Motion of the human mandibular condyle during mastication. J Dent Res 2001; 80: 437-442.

9. Bando E, Nakano M, Fujimura T, et al. Clinical examination of stomatognathic function - Using digital type jaw movement analyzer MM-JI-. J Jpn Prosthodont Soc 1998; 42: 894-901.

10. Lauritzen AG, Wolford LW. Occlusal relationships: The split-cast method for articulator techniques. J Prosthet Dent 1964; 14: 256-265.

11. Lauritzen AG.. Atlas of occlusal analysis. Colorado Springs: HAH Publications, 1974.

12. Aull AE. Condylar determinants of occlusal patterns. J Prosthet Dent 1965; 15: 826-849. 